\title{
The development and inflammatory features of radiotherapy-induced glossitis in rats
}

\author{
Chun-Yang Li ${ }^{1}$, Xiao-Hua Chen ${ }^{2}$, Xiao-An Tao ${ }^{3}$, Juan Xia $^{3}$, Bin Cheng ${ }^{3}$
}

\begin{abstract}
${ }^{1}$ Ph.D, Department of Stomatology, The Fifth Affiliated Hospital, Sun Yat-sen University, Zhuhai 519000, China
${ }^{2}$ M.M.S, Department of Oral Pathology, The Guanghua School of Stomatology/Affiliated Hospital of Stomatology, Sun Yat-sen University, Guangzhou 510060, China

${ }^{3}$ Ph.D, Department of Oral Medicine, The Guanghua School of Stomatology/Affiliated Hospital of Stomatology, Sun Yat-sen University, Guangzhou 510060, China
\end{abstract}

Correspondence:

Department of Oral Medicine,

The Guanghua School of Stomatology/Affiliated Hospital of Stomatology, Sun Yat-sen University, 56\# Lingyuan Road West, Guangzhou 510060, China

chengbin@mail.sysu.edu.cn

Received: 04/06/2010

Accepted: $14 / 11 / 2010$

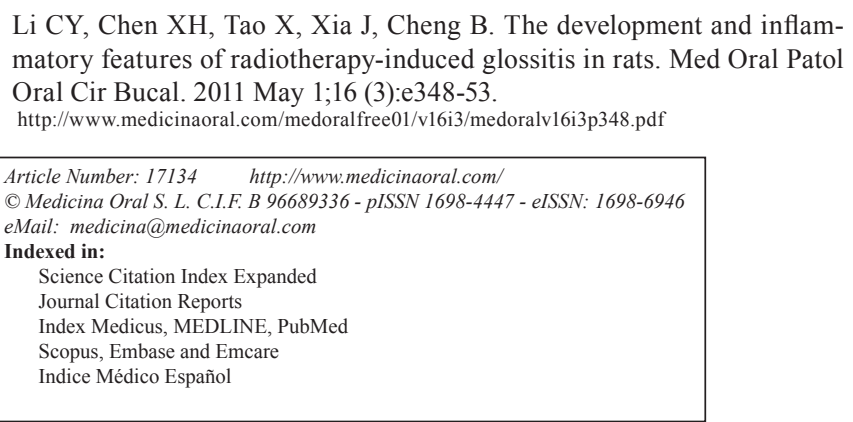

\begin{abstract}
Objectives: To improve the existing animal models (mice, rats, and hamsters) for radiotherapy-induced oral mucositis (RTOM), thereby establishing a radiotherapy-induced glossitis (RTG) Sprague-Dawley (SD) rat model. Study Design: A lead device was designed to limit radiation exposure to a $1 \times 1 \mathrm{~cm} 2$ area of a rat's dorsal anterior tongue with a single $30 \mathrm{~Gy}$ of X-ray radiation. The general conditions of the irradiated rats, such as body-weight and behavior, were observed. The oral mucositis index (OMI) of the RTG rats were measured daily. Histological changes of the irradiated tongue tissues were assayed by H\&E staining.

Results and Conclusion: No significant changes were clinically observed 3 to 4 days after irradiation. At 5 to 6 day, punctuation and confluenced redness of the mucosa were observed. The small blood vessels became more extensive, engorged, thin vessel walls. More infiltrating cells were observable, necrosis and exfoliation of the squamous cells appeared, and the formation of an ulcerative lesion could be observed. Seven to 15 days, the exfoliated epithelial layer was observed to have formed an ulcerative lesion, then aggravated ulcerative lesions consisting of pseudomembranous filament exudates could be observed. The structure of the epithelium had become completely disintegrated, forming deep, microscopic ulcerative lesions. Twenty-one days, the periphery of the ulcer was observed to have begun to heal, and granulation tissue could be observed at the bottom of the ulceration. At 35 days after irradiation, the epithelial structure presented again, but the epithelium was very thin. An RTG animal model was successfully established in SD rats, which provides a new research platform for the study of RTOM pathogenesis.
\end{abstract}

Key words: Radiotherapy, oral mucositis, glossitis. 


\section{Introduction}

Radiotherapy-induced oral mucositis (RTOM) is one of the most debilitating side effects of radiotherapy, and threatens the effectiveness of antitumor therapy because it limits the dose of applied radiation, causes malnutrition and weight loss, decreases the patient's quality of life, increases the cost of healthcare, prolongs the length of in-patient stays, and demands more medical and social resources (1). Therefore, RTOM is the primary dose-limiting factor in head and neck malignancy radiotherapy, which is a problem that requires immediate resolution $(2,3)$.

Presently, only one agent, Palifermin (recombinant keratinocyte growth factor-1) (4), is approved for the treatment of cancer treatment-related oral mucositis, although sev $\neg$ eral others are at various stages of development (5-10). The pathogenesis of RTOM is not fully understood and prophylactic and/or therapeutic strategies should be evaluated with further investigation (11).

Several animal models have been used to investigate RTOM. In the 1980s, mouse lip $(12,13)$ and mouse ventral tongue mucosa (14) were used as animal models for RTOM, whereas in the 1990s, the hamster cheek pouch model was developed (15). Dose response curves for the appearance of gross ulceration in these tissues following irradiation with single and fractionated regimens have been obtained (14-18), identifying several flaws in the aforementioned animal models. The first flaw in these models is the high animal death rate, particularly the mice used in the mouse model. When the mouse snout is irradiated in the mouse model, the brain is inevitably also irradiated, frequently resulting in death of the experimental animals. A second flaw includes the use of multiple instances of anesthesia, irradiation, and uncontrollable fractional irradiation, such that homogenous tissue irradiation is difficult to obtain, sometimes resulting in animal death. A third flaw of the aforementioned models is that the usable quantity of irradiated mouse oral tissue is small, limiting extensive analysis. A final flaw in the aforementioned animal models is that although buccal pouch eversion provides sufficient oral tissue for investigation and is easily isolated from the rest of the body during radiation treatment, the hamster buccal pouch is an immune-privileged site, and cannot therefore provide an accurate immune response reaction.

The present study intends to duplicate and further improve existing RTOM animal models by only exposing anterior dorsal tongue mucosal tissue to metered, single 30-Gy doses of X-rays and to develop an animal model that effectively represents radiotherapy-induced glossitis (RTG) in SD rats. Furthermore, this study intends to provide a new research platform to investigate prophylactic and therapeutic methods for RTOM.

\section{Materials and Methods}

\section{-Ethics}

All procedures in this animal study were reviewed and approved by the Animal Ethics Committees at the Institute of Medical and Veterinary Sciences, Sun Yat-sen University, Guangzhou, China. The work and animal care conducted in this study comply with the National Health and Medical Research Council Code of Practice of Care for Animals in Research and Training (1998). -Animals and housing

Forty-eight specific pathogen-free (SPF) male SpragueDawley (SD) rats, aged 10 to 12 weeks and weighing 250 to $300 \mathrm{~g}$, were purchased from the Northern Campus Animal Center of Sun Yat-sen University [the certification number of the experimental animals was SCXK (Guangdong) 2004-0011; approved certification number: 2005A060].

The rats were housed in polycarbonate cages with six rats per cage, and were provided free access to standard rat food and filtered city tap water from standard perspex drinking bottles. The rats were housed with a 12-hr light/12-hr dark cycle (the light phase ranged from 06:00 to 18:00 at a lux of 150 to 300) in a room maintained at a temperature of $23 \pm 1{ }^{\circ} \mathrm{C}$ and humidity of $55 \pm 5 \%$ under specified pathogen-free conditions.

\section{-Irradiation Protocol and Experimental Design}

All of the rats used in this study were allowed to adapt to the housing conditions for two to three days prior to $\mathrm{X}$-ray irradiation in order to mitigate adverse irradiation responses. The experimental rats were randomly divided into eight groups of six, with one group as the control and the remaining seven as experimental groups.

Prior to X-ray irradiation, each rat received an abdominal injection of $6 \mathrm{ml} / \mathrm{kg}$ of a $5 \%$ chloral hydrate solution. The 6 rats in the control group were shielded under a lead plate, whereas the 42 rats in the remaining seven experimental groups were irradiated with no protective shielding. Exclusive irradiation of each rat's anterior dorsal tongue was achieved by using a $2 \mathrm{~mm}$-thick, cone-shaped lead shielding device that was specially designed and constructed in our laboratory. The tongue of every rat was guided through a notch in the lead device using forceps such that the anterior dorsal part of the tongue could be fixed to the outer surface of the lead device using adhesive tape. An accessory lead plate with a "u-shaped" notch was used to cover the tongue and limit the irradiated area to $10 \times 10 \mathrm{~mm} 2$. These two lead plate layers ensured that the rest of the animal's body was shielded from radiation exposure (Fig. 1).

A deep X-ray machine, (type F34-I; Dong Fang, Beijing, China) using an acceleration voltage of $210 \mathrm{kV}$, working current of $12 \mathrm{~mA}$, a target distance of $40 \mathrm{~cm}$, an irradiation field of $10 \times 15 \mathrm{~cm} 2$, a 4-mm aluminum filter system, and a delivered dosage of $100.75 \mathrm{cGY} / \mathrm{min}$ was used to irradiate the rats used in this study. The heads of six rats were placed in the same irradiation field during radiation 

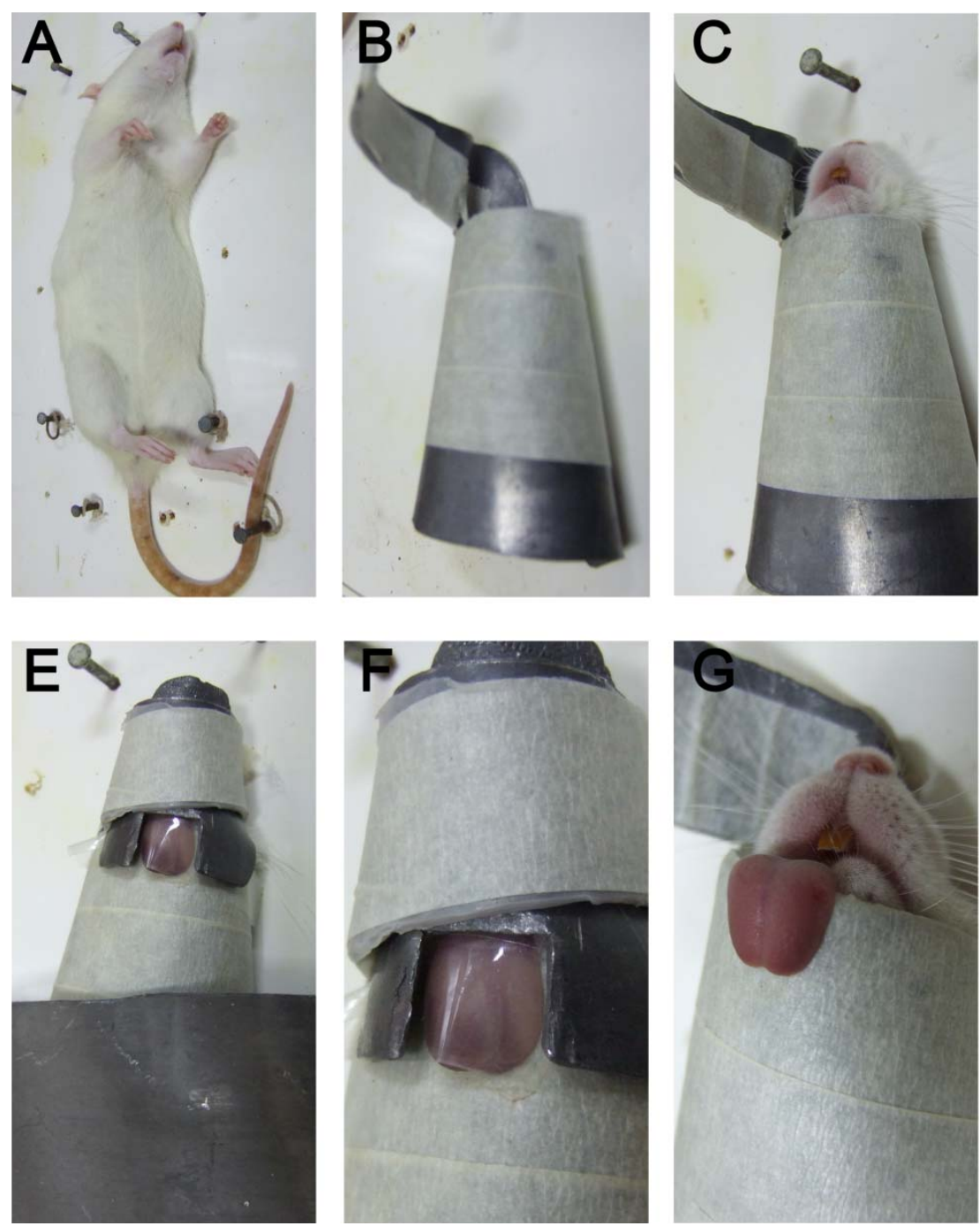

Fig. 1. Irradiation scope and radiation protection of the experimental rats. A. An anesthetized rat. B. The $2 \mathrm{~mm}$-thick, cone-shaped lead device designed by our research team. C, D, E, and F. The tongue of every rat was guided through a notch in the lead device using forceps such that the anterior dorsal part of the tongue could be fixed to the outer surface of the lead device using adhesive tape. An accessory lead plate with a "u-shaped" notch was used to cover the tongue and limit the irradiated area to $10 \times 10 \mathrm{~mm} 2$. These two lead plate layers ensured that the rest of the animal's body was shielded from radiation exposure. G. The anterior dorsal tongue after irradiation.

exposure. Thirty Gray of radiation were delivered at one time to all of the experimental rats in a group at 09:00.

-Gross observation and data sampling frequency

All rats were carefully monitored twice or four times daily throughout the experimentation period. Each rat was weighed at 08:00, had their feces inspected, and behavioral characteristics assessed daily. Two cotton threads were used to open the rat's oral cavity, the tongue was gently pushed back using a cotton ball, and digital images of the irradiated tongue tissue were taken using a Fuji digital camera (Model: E900) under a cold lamp source. Clinical record sheets were prepared according to observations of the exposed tongue. The dorsal tongue surface of each rat was evaluated using a previously developed method by Parkins [12] (Table 1).
Table 1. The oral mucositis scoring system used in this study and originally proposed by Parkins [12].

\begin{tabular}{|c|l|}
\hline Score & \multicolumn{1}{|c|}{ Description } \\
\hline 0 & Normal \\
\hline 0.5 & Slightly pink \\
\hline 1.0 & Slightly red \\
\hline 2.0 & Severe reddening \\
\hline 3.0 & Focal desquamation \\
\hline 4.0 & $\begin{array}{l}\text { Exudation covering less than one-half of the } \\
\text { irradiated mucosae }\end{array}$ \\
\hline 5.0 & $\begin{array}{l}\text { Virtually complete ulceration } \\
\text { of the mucosae }\end{array}$ \\
\hline
\end{tabular}


A

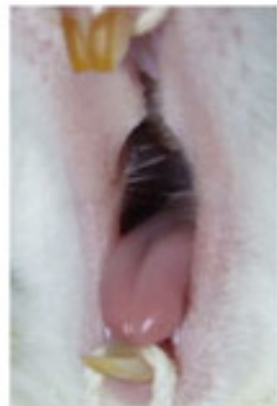

$\mathrm{C}$

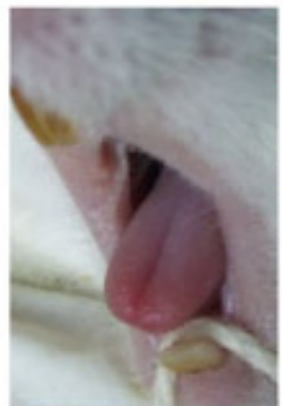

E

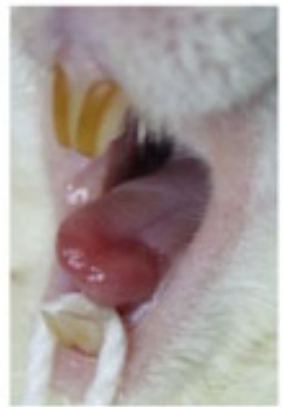

G
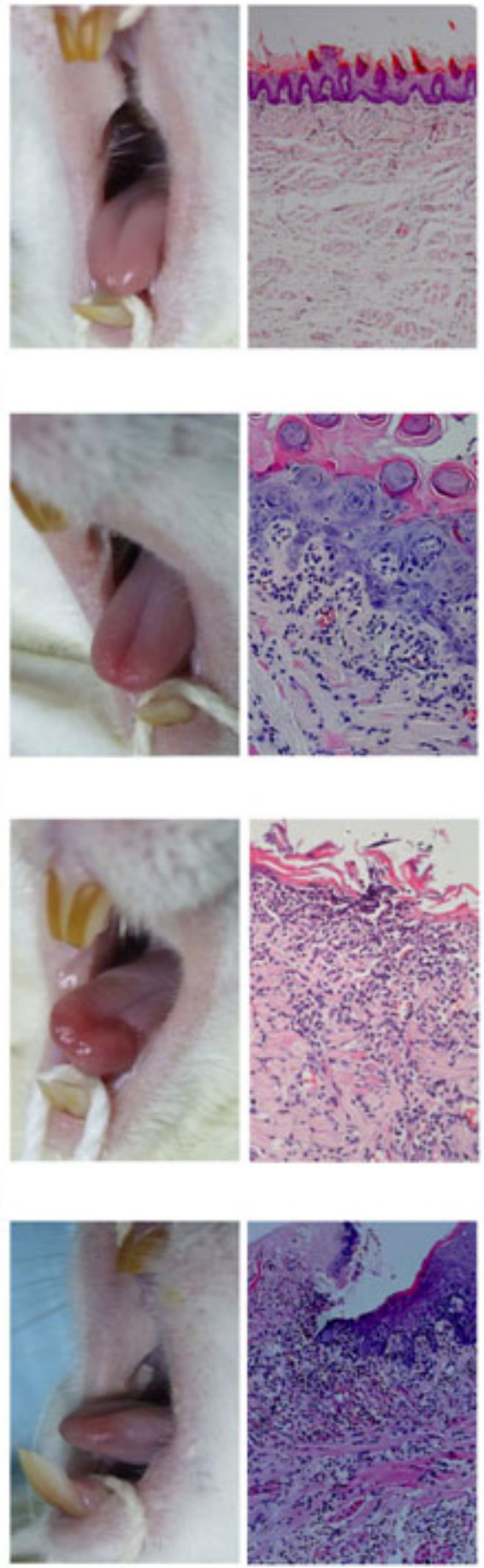

B
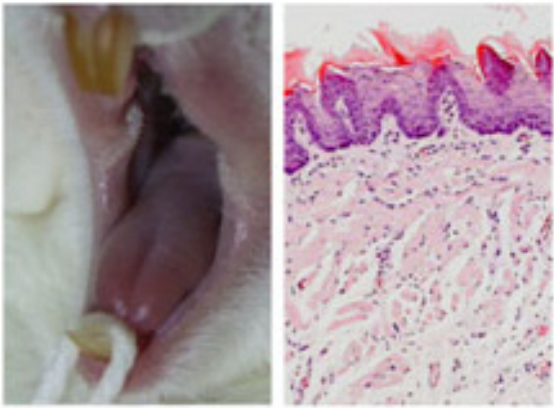

D
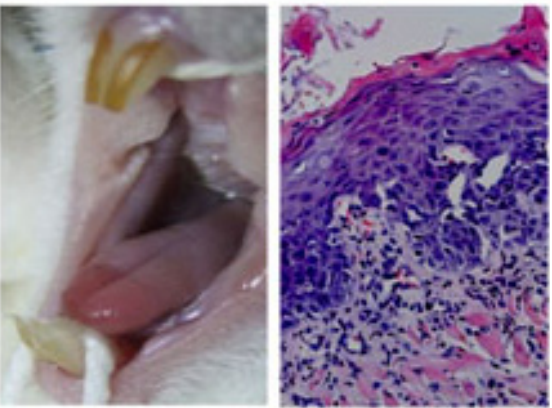

$\mathrm{F}$
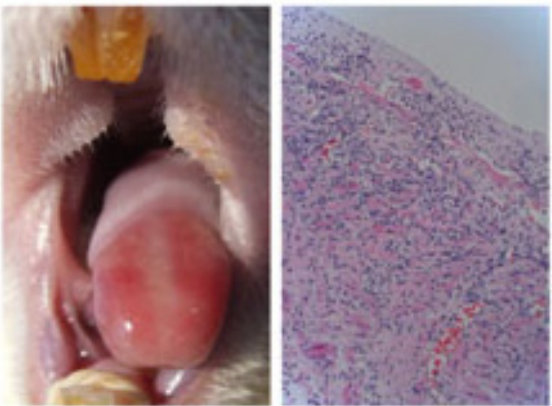

$\mathrm{H}$

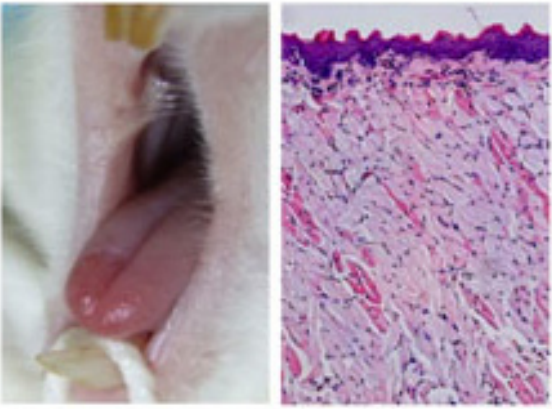

Fig. 2. Macroscopic (left panel) and microscopic (right panel) images of anterior dorsal rat tongues after exposure to a single 30-Gy dose of X-ray radiation. A. Normal control. B. Three days after irradiation. C. Five days after irradiation. D. Eight days after irradiation. E. Fourteen days after irradiation. F. Twenty-one days after irradiation. G. Twenty-eight days after irradiation. H. Thirty-five days after irradiation. 


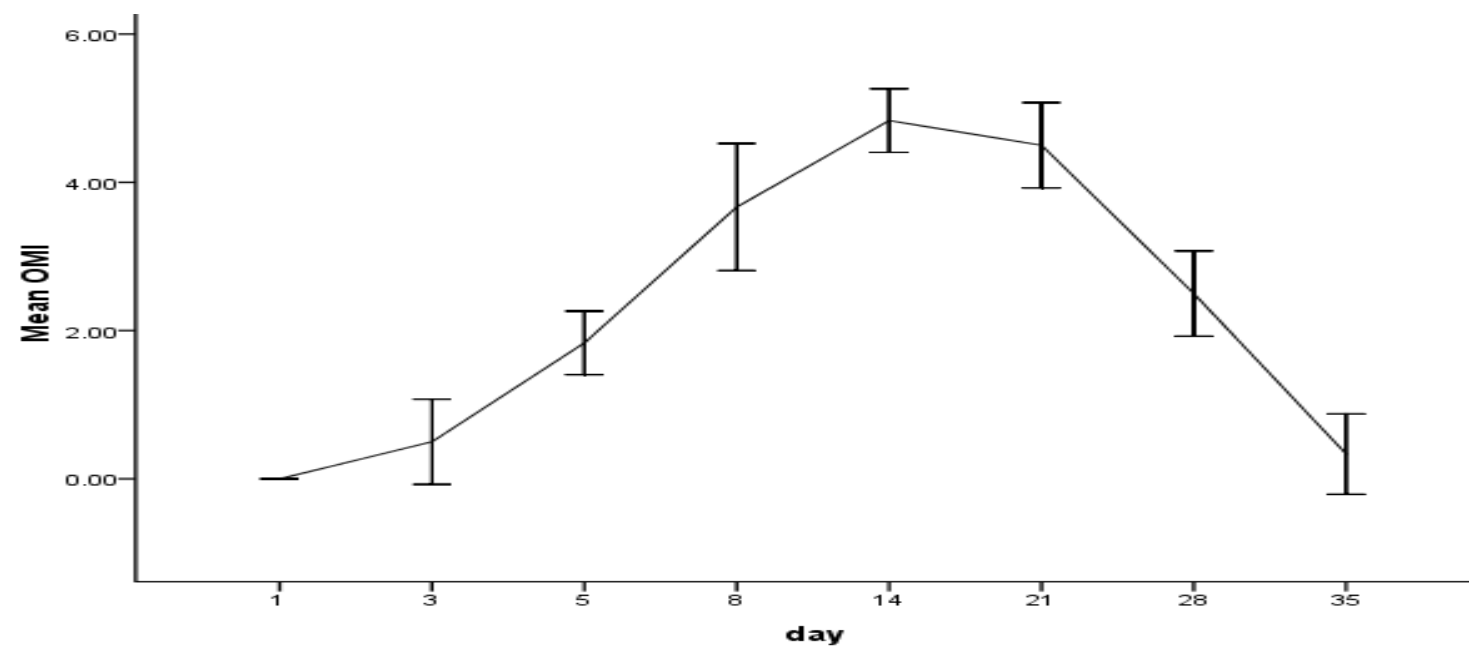

Fig. 3. Mucositis curve following irradiation of anterior dorsal tongue mucosae in SD rats. Dorsal tongue Symptoms were observed at $1,3,5,8,14,21,28$, and 35 day after irradiation, which were classified using the 0 -to- 5 scale described in the text. The error bars indicate the standard error of the mean ( $\mathrm{n}=6$ at each time point).

OMI were expressed as mean \pm SD.

-Histology (H\&E staining)

Three, 5, 8, 14, 21, 28, and 35 days after irradiation, six rats randomly selected from each group were exsanguinated via a cardiac puncture and then killed via cervical dislocation. Tongue specimens were obtained by cutting the tongue root, which were then bathed in a neutral buffered $10 \%$ concentrated formalin solution, paraffin embedded, and excised for hematoxylin and eosin staining. Histological assessment was conducted using optical microscopy.

\section{Results}

\section{-Survival rate}

No rats accidently died during the 6-week experimental period.

-Anterior dorsal tongue mucosal macroscopical and histological analysis

Four days after irradiation, no significant changes in the irradiated mucosae were observed (Fig.2.B). Five to 6 days after irradiation, reddening and red spots were observed in the anterior tongue mucosae of the irradiated rats (Fig.2.C left). The histology in that section showed that the small blood vessels became more extensive, engorged, thin vessel walls. More infiltrating cells were observable, necrosis and exfoliation of the squamous cells appeared, and the formation of an ulcerative lesion could be observed (Fig.2.C right). Seven to 8 days after irradiation, the tongue mucosa of each irradiated rat exhibited discernible ulcers (Fig.2.D left). Nine to 14 days after irradiation, the previously observed ulcers fused together into a laminated form resulting in the formation of ulcers with large surface areas (Fig.2.E left). Fifteen to 17 days after irradiation, mitigated lam- inated-form ulcers could be observed. The structure of the epithelium had become completely disintegrated, forming deep, microscopic ulcerative lesions (Fig.2.D right, 2.E right). Eighteen days after irradiation, a consistent and gradual ulcer reduction was observed. Twenty days after irradiation, the irradiated anterior tongue mucosa was generally healed (Fig.2.F left, 2.G left), and granulation tissue could be observed at the bottom of the ulceration (Fig.2.F right, 2.G right). It was observed to be completely healed 15 days later (Fig.2.H left), with no visible differences in comparison to the control mucosae (Fig.2.A left), but the epithelium was very thin (Fig.2.H right).

-Mucositis Index

Based on the evaluations described above, a typical mucositis curve was obtained following the single 30-Gy irradiation regime (Fig. 3).

\section{Discussion}

Tongue ulceration represents the mucosal damage seen in high-grade oral mucositis in cancer patients $(1,5)$. The present study was designed to identify all possible alterations in tongue mucosae due to locally applied irradiation. Radiotherapy-induced oral mucositis animal models have been described previously (12-18); however, these models have frequently used mice, and it is technically difficult to produce definitive irradiation doses in mouse buccal or gingival mucosa due to the small size of the typical mouse. Therefore, we have selected the tongue as a more appropriate irradiation site. Furthermore, an SD rat anterior tongue mucosal mucositis model was preferred for this study, since it provides the best reproducibility, adequate space for 
sufficient tissue sampling, and definitive irradiation features, such as voltage, distance, and exclusive irradiation of the anterior tongue mucosae via appropriate shielding. Additionally, the dorsal surface of the tongue can be covered with keratinized epithelium and nonkeratinized epithelium and can adequately express the epithelium tissues of oral mucosae. In comparison to previous radiotherapy-induced oral mucositis animal models, the tongue can be easily observed on a daily schedule $(5,14,19)$.

In this study, we designed a 2-mm-thick, cone-shaped lead device that limited irradiation to a $1 \times 1 \mathrm{~cm} 2$ area of anterior dorsal tissue, while the remainder of the rat body was effectively shielded from radiation (see Fig. 1 for an illustration). In contrast, snout-only irradiation of mice in mouse models is not sufficiently specific as to obviate the ultimate cause of death of irradiated animals. In the present model, a single instance of anesthesia and irradiation was employed to mitigate the previously observed high rates of experimental animal death. No accidental animal death was observed in the rat model developed in this study, despite that the irradiation dose of 30 Gy used herein is identical to that used in previous reports (5). The typical appearance and progression of oral mucositis were observed to occur at the local region of the anterior dorsal tongue of the irradiated rats, which appeared to be in a latent stage 1 to 4 days after irradiation, present gradual ulceration 5 to 14 days after irradiation, peak in ulcerification 15 to 20 days after irradiation, begin to gradually heal 15 days after irradiation, and be completely healed 35 days after irradiation. Most importantly, flash anesthesia was not needed for daily observation of the irradiated tissue when using the present model.

In preliminary studies, we demonstrated the correlation of radiation dosage to the degree of tongue mucosal damage and determined that a single fraction of high-level radiation at 30 Gy directly delivered to the rat anterior dorsal region reliably resulted in overt tongue mucosal ulceration that peaked 9 to 14 days after irradiation (data not shown). In order to assess the severity of mucositis in the mouse or hamster cheek pouch models, the animals typically need to be anesthetized and their buccal pouches everted[15]; however, in the present model, it much more facile to directly observe the anterior and dorsal regions of the rats' tongues without the need of anesthesia. Taken together, the present model adequately describes the clinical features of severe oral mucositis and provides an easily observable area of ulceration.

\section{Conclusion}

The RTG model developed herein is a simple, but effective animal model that describes the pathogenesis of RTOM. This model was successfully established in SD rats, which provides a new research platform to investigate prophylactic and therapeutic methods of RTOM.

\section{References References with links to Crossref-DOI}

1. Sciubba JJ, Goldenberg D. Oral complications of radiotherapy. Lancet Oncol. 2006;7:175-83.

2. Keefe DM, Schubert MM, Elting LS, Sonis ST, Epstein JB, RaberDurlacher JE, et al. Updated clinical practice guidelines for the prevention and treatment of mucositis. Cancer. 2007;109:820-31.

3. Rosenthal DI, Trotti A. Strategies for managing radiation-induced mucositis in head and neck cancer. Semin Radiat Oncol. 2009;19:2934.

4. Spielberger R, Stiff P, Bensinger W, Gentile T, Weisdorf D, Kewalramani $\mathrm{T}$, et al. Palifermin for oral mucositis after intensive therapy for hematologic cancers. N Engl J Med. 2004;351:2590-8.

5. Zhao J, Kim KA, De Vera J, Palencia S, Wagle M, Abo A. R-Spondin1 protects mice from chemotherapy or radiation-induced oral mucositis through the canonical Wnt/beta-catenin pathway. Proc Natl Acad Sci U S A. 2009;106:2331-6.

6. Haagen J, Krohn H, Röllig S, Schmidt M, Wolfram K, Dörr W. Effect of selective inhibitors of inflammation on oral mucositis: preclinical studies. Radiother Oncol. 2009;92:472-6.

7. Ara G, Watkins BA, Zhong H, Hawthorne TR, Karkaria CE, Sonis ST, et al. Velafermin (rhFGF-20) reduces the severity and duration of hamster cheek pouch mucositis induced by fractionated radiation. Int J Radiat Biol. 2008;84:401-12.

8. Wu HG, Song SY, Kim YS, Oh YT, Lee CG, Keum KC, et al. Therapeutic effect of recombinant human epidermal growth factor (RhEGF) on mucositis in patients undergoing radiotherapy, with or without chemotherapy, for head and neck cancer: a double-blind placebo-controlled prospective phase 2 multi-institutional clinical trial. Cancer. 2009;115:3699-708.

9. Arora H, Pai KM, Maiya A, Vidyasagar MS, Rajeev A. Efficacy of $\mathrm{He}-\mathrm{Ne}$ Laser in the prevention and treatment of radiotherapyinduced oral mucositis in oral cancer patients. Oral Surg Oral Med Oral Pathol Oral Radiol Endod. 2008;105:180-6.

10. Murphy CK, Fey EG, Watkins BA, Wong V, Rothstein D, Sonis ST. Efficacy of superoxide dismutase mimetic M40403 in attenuating radiation-induced oral mucositis in hamsters. Clin Cancer Res. 2008;14:4292-7.

11. Sonis ST. The pathobiology of mucositis. Nat Rev Cancer. 2004;4:277-84.

12. Parkins CS, Fowler JF, Yu S. A murine model of lip epidermal/ mucosal reactions to X-irradiation. Radiother Oncol. 1983;1:159-65.

13. Xu FX, van der Schueren E, Ang KK. Acute reactions of the lip mucosa of mice to fractionated irradiations. Radiother Oncol. 1984;1:369-74.

14. Moses R, Kummermehr J. Radiation response of the mouse tongue epithelium. Br J Cancer Suppl. 1986;7:12-5.

15. Sonis ST, Tracey C, Shklar G, Jenson J, Florine D. An animal model for mucositis induced by cancer chemotherapy. Oral Surg Oral Med Oral Pathol. 1990;69:437-43.

16. Dörr W, Emmendörfer H, Haide E, Kummermehr J. Proliferation equivalent of 'accelerated repopulation' in mouse oral mucosa. Int J Radiat Biol. 1994;66:157-67.

17. Dörr W, Kummermehr J. Accelerated repopulation of mouse tongue epithelium during fractionated irradiations or following single doses. Radiother Oncol. 1990;17:249-59.

18. Dörr W, Kummermehr J. Proliferation kinetics of mouse tongue epithelium under normal conditions and following single dose irradiation. Virchows Arch B Cell Pathol Incl Mol Pathol. 1991;60:28794.

19. López-Castaño F, Oñate-Sánchez RE, Roldán-Chicano R, Cabrerizo-Merino MC. Measurement of secondary mucositis to oncohematologic treatment by means of different scale. Review. Med Oral Patol Oral Cir Bucal. 2005;10:412-21. 